\title{
COMPARANDO AS CONSOANTES DAS LÍNGUAS TAPAJÚNA E SUYÁ
}

\author{
Cíntia Karla Coelho RODRIGUES* \\ Marília de Nazaré FERREIRA-SILVA**
}

- RESUMO: Este estudo faz considerações sobre a investigação histórico-comparativa da língua tapajúna (Jê), mais especificamente sobre as consoantes em seu aspecto fonéticofonológico, comparando cognatos dessa língua e da língua suyá (também da família Jê) com o objetivo de verificar suas semelhanças e diferenças no contexto das consoantes, tendo em vista contribuir para a reconstrução do tronco linguístico Macro-Jê. Para o estudo aqui desenvolvido, foi utilizado o método comparativo por meio do qual são verificadas as correspondências existentes entre as línguas envolvidas em um estudo dessa natureza, com o fim de observar como seria possivelmente constituída a forma original, que teria originado as formas das duas línguas. Os dados de tapajúna e de suyá também foram comparados aos dados do Proto-Jê, reconstruído por Davis (1966). De um modo geral, observa-se que há poucas diferenças entre tapajúna e suyá. No caso das variações em contextos fonéticofonológicos idênticos, há a questão da variação de faixa etária, como a ocorrência das consoantes pré-nasalizadas na fala dos mais jovens do povo tapajúna, enquanto os mais velhos usam as nasais.

- PALAVRAS-CHAVE: Método histórico-comparativo. Tapajúna. Suyá. Aspectos fonéticofonológicos. Consoantes. Cognatos.

\section{A língua Tapajúna}

A língua tapajúna pertence à família Jê falada por cerca de 40 indivíduos. ${ }^{1} \mathrm{O}$ povo tapajúna, até o momento de realização da pesquisa que embasou o presente trabalho, vivia na aldeia Metyktire juntamente com os Mebengôkre (Kayapó), nas imediações do Parque Indígena do Xingu, no estado do Mato Grosso, no Brasil Central. Devido a esse reduzido número de falantes e por se encontrar em uma situação de atrito linguístico (já que os tapajúna viviam em meio à predominância da língua e dos costumes dos kayapó, além de sofrerem a influência da língua portuguesa), a língua tapajúna corre risco de extinção.

\footnotetext{
* Secretaria de Educação do Estado do Pará. Belém - PA - Brasil. 66.075-100 -cintiacoro@gmail.com

** UFPA - Universidade Federal do Pará. Instituto de Letras e Comunicação - Faculdade de Letras. Belém -PABrasil. 66.075-100 - marilia@ufpa.br

1 Censo realizado por Marília Ferreira em 2005 na aldeia Mebengôkre, no estado do Mato Grosso.
} 
O tapajúna não conta com estudos descritivos detalhados. Há somente dois trabalhos de cunho linguístico publicados até o momento: o trabalho de Seki (1989), que se constitui em um estudo diacrônico entre dados do tapajúna e do suyá, comparando-os aos dados do Proto-Jê reconstruídos por Davis (1966);2 e o trabalho de Santos (1997), o qual consiste em uma descrição dos aspectos morfossintáticos do suyá. Já em 2010, foi defendida, na Unicamp, a dissertação de mestrado de Nayara Camargo intitulada Língua Tapayúna: aspectos sociolinguísticos e uma análise fonológica preliminar. Em novembro de 2004, a língua tapajúna começou a ser estudada e descrita por Marília Ferreira e, por meio das coletas de dados e estudos desenvolvidos pela pesquisadora até 2005, foi possível realizar uma análise descritiva e diacrônica dos aspectos fonéticofonológicos da língua, a qual foi apresentada como dissertação de mestrado em Letras ao Programa de Pós-Graduação em Letras da Universidade Federal do Pará, em 2007. O objetivo do trabalho foi contribuir para o refinamento da reconstrução histórica do tronco Macro-Jê, o qual é bem menos conhecido que o Tupi, por exemplo.

O tapajúna é uma língua muito próxima do suyá, sendo essas línguas muito semelhantes estruturalmente, devido à genética delas - ambas pertencem à família Jê, do tronco Macro-Jê. Pode-se ouvir afirmações sobre o fato de o tapajúna ser um dialeto do suyá, com base nos depoimentos dos próprios falantes. Entre as semelhanças existentes entre essas línguas, podem-se citar: a ocorrência dos prefixos relacionais, a ocorrência dos verbos em posição final de sentença, um inventário sonoro típico de línguas Jê, em que as vogais se sobrepõem às consoantes, entre outras.

A urgência em estudar o tapajúna se deve ao fato de essa língua estar seriamente ameaçada de extinção. Nosso propósito é apresentar evidências que contribuam para a reconstrução do tronco linguístico Macro-Jê por meio da análise histórico-comparativa dos aspectos fonético-fonológicos da língua tapajúna, considerando-se, no caso deste artigo, especificamente os segmentos consonantais, comparando-os aos dados do suyá, face aos dados reconstruídos por Davis (1966) para o Proto-Jê.

\section{Considerações sobre a Linguística Histórica}

Desde a publicação do Curso de Linguística Geral, de Ferdinand de Saussure, em 1913, tem-se a distinção entre os estudos sincrônicos e os estudos diacrônicos. A Linguística Histórica se ocupa da análise diacrônica de línguas com o objetivo de analisar os processos de mudança sofridos por elas através dos tempos.

Nosso objetivo não é a comparação do presente trabalho com o de Seki (1989). 
Gabas Junior (2001, p.78) afirma que, com os estudos desenvolvidos a partir do século XIX, sobre as línguas latim, grego e sânscrito, a linguística buscou explicar as aparentes semelhanças entre as diferentes línguas humanas.

Semelhanças aparentes entre línguas distintas sempre chamaram a atenção de estudiosos e curiosos em todo o mundo. Foi, no entanto, apenas no final do século XVIII que Sir William Jones, um juiz inglês da Índia, oficial e acertadamente propôs que o latim, o grego e o sânscrito eram línguas aparentadas entre si. Sir Jones foi além: não apenas demonstrou com os métodos de correspondência de som, que tal afirmação era possível de ser comprovada, como também hipotetizou que as três línguas eram derivadas de uma outra língua, possivelmente já extinta (hoje sabemos que se trata do protoindo-europeu). Era o começo de estudos sistemáticos em Linguística Histórica e Comparativa, que, graças à farta quantidade de registros históricos, se concentraram nas línguas indo-europeias.

Como se pode perceber, estudos históricos de línguas vêm sendo desenvolvidos há bastante tempo e têm sido de grande importância para os avanços da linguística. De acordo com Faraco (1991, p.9), "A realidade empírica central da linguística histórica é o fato de que as línguas humanas mudam com o passar do tempo". Isso significa que as línguas não se constituem em algo estático, muito pelo contrário, sofrem mudanças no tempo. Há mudanças que podem ser percebidas no momento em que ocorrem, no entanto, há outras que só o tempo é capaz de torná-las evidentes. É nesse ponto que encontramos um dos papéis fundamentais da linguística histórica. Além disso, temos que reconhecer a importância dessa área para os estudos antropológicos e arqueológicos.

Os estudos históricos das línguas se constituem em fonte importantíssima para se compreender a cultura e o modo de viver dos antepassados que as falavam. Por exemplo, conforme Ribeiro (em comunicação pessoal, em 2005), a partir dos estudos comparativos de línguas da família Jê, está sendo possível reconstruir termos que se referem à cultura material, o que permite obter informações sobre a cultura do povo que falava a língua que originou as línguas Jê.

Crowley (1997) considera que compreender cultura por meio da Linguística Histórica é realmente possível, pois com a análise comparativa, podemos descobrir como era a forma de ver o mundo das populações ancestrais, isto é, por meio do estudo de uma língua, descobre-se a cultura do povo que a fala ou falava, já que a língua é, em si, cultural e expressa os dados e elementos culturais de determinada sociedade.

Na próxima seção serão apresentados os aspectos fonético-fonológicos do tapajúna, com vistas à reconstrução do Proto-Jê. 


\section{Aspectos fonético-fonológicos do tapajúna com vistas à reconstrução do Proto-Jê}

De acordo com as análises que vêm sendo desenvolvidas e com as observações dos dados do Proto-Jê, reconstruídos por Davis (1966), o tapajúna apresenta muitas semelhanças com o Proto-Jê e algumas mudanças em alguns segmentos fonético-fonológicos de certos dados aqui apresentados.

Segundo Ribeiro (2004, p.91), "Davis (1966) reconstrói um inventário fonológico e um vocabulário Proto-Jê", o que pode ser verificado parcialmente no quadro 1, com acréscimo de dados da língua tapajúna.

\begin{tabular}{|c|c|c|c|c|c|c|c|}
\hline & Proto-jê & Apinajé & Canela & Suyá & Xavante & Kaingáng & Tapajúna \\
\hline 1.dente & cwa & wacwa & iicwa & iitwa & ?wa & jã & twa \\
\hline 2.fogo & kuzi & kuwi & kuhi & $\mathrm{k}^{\mathrm{h}} \mathrm{usi}$ & ?uni & & kuti \\
\hline 3.carne & jĩ & $\tilde{1}, \ldots$ nĩ & hĩ & nĩ & jĩ & nĩ & jĩ \\
\hline 4.comida & nо̃ & $\tilde{\text { o, }}$, по & & & กо̃ & $\begin{array}{l}\text { yẽ } \tilde{n} \text { 1com } \\
\text { er } \|\end{array}$ & ก $\tilde{}$ \\
\hline $\begin{array}{l}\text { 5. asa, } \\
\text { pena }\end{array}$ & zạra & $\begin{array}{l}\text { Para, } \\
\text { jara }\end{array}$ & haaraa & saara & $\begin{array}{l}\text { necre } \\
\text { thair\| }\end{array}$ & $\phi \tilde{e} r \tilde{e}$ & øูara \\
\hline
\end{tabular}

Quadro 1 - Dados comparativos entre línguas Jê e o Proto-Jê

Fonte: Autoria própria.

Observa-se que há muitas semelhanças fonológicas entre suyá e tapajúna e leves mudanças ocorridas nas duas línguas com relação aos dados do Proto-Jê. No dado 1, por exemplo, suyá e tapajúna têm a mesma forma para dente, tendo havido uma mudança em relação ao Proto-Jê no protofonema inicial /*c/ que é refletido como /t/ nas duas línguas, o que nos leva a dizer que apenas mudou o ponto de articulação, passando de oclusiva palatal surda para oclusiva alveolar surda.

O quadro 1, como é possível observar, mostra algumas reconstruções feitas por Davis (1966), nas quais compara itens lexicais de algumas línguas Jê. Conforme a distribuição das línguas, podemos perceber que o kaingáng sofreu mais alterações que as outras línguas. As demais línguas são muito mais próximas entre si. Para este estudo, no entanto, apenas observaremos as consoantes do suyá e do tapajúna, que são línguas, de acordo com os dados apresentados, proximamente relacionadas, diferenciando-se somente em alguns aspectos.

Sendo assim, veremos a partir deste momento algumas análises feitas quanto à relação entre essas duas línguas em comparação ao Proto-Jê, tendo em vista alguns segmentos consonantais, a saber, os que refletem os seguintes protofonemas: /* $\mathrm{p} /, /{ }^{*} \mathrm{z} /, /{ }^{*} \mathrm{c} / \mathrm{e} /{ }^{*} \mathrm{~m} /$. 


\section{Semelhanças e diferenças com relação ao Proto-Jê}

Tanto o suyá quanto o tapajúna apresentam características fonético-fonológicas muito semelhantes às do Proto-Jê, o que contribui muito para o fato de considerar o Macro-Jê como tronco linguístico. Isso pode ser verificado no quadro 2 :

\begin{tabular}{|c|c|c|c|c|}
\hline Protofonema & Proto-Jê & Tapajúna & Suyá & Tradução \\
\hline$*_{\pi}$ & 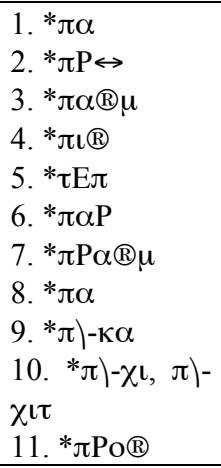 & $\begin{array}{l}\eta \omega \alpha \\
\eta \mathrm{P} \alpha \\
\mu \leftrightarrow \circledR \mu \\
\eta \omega \iota \AA \\
\tau \mathrm{E} \omega \mathrm{E}, \tau \mathrm{E} \pi \\
\eta \omega \alpha \varphi \\
\eta \mathrm{P} \alpha{ }^{\circledR} \mu \\
\omega \alpha \\
\eta \omega \mid \kappa \alpha \\
\omega \mid \tau \iota \\
\eta \mathrm{Po} \AA \mu\end{array}$ & 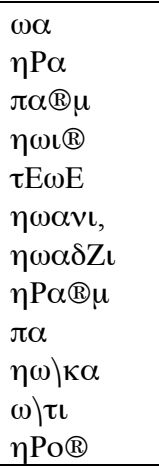 & $\begin{array}{l}\text { 'braço' } \\
\text { 'cinzas' } \\
\text { 'pai' } \\
\text { 'lenha' } \\
\text { 'peixe' } \\
\text { 'pé' } \\
\text { 'fome' } \\
\text { 'eu' } \\
\text { 'terra' } \\
\text { 'um' } \\
\text { 'esposa' }\end{array}$ \\
\hline$* \xi$ & 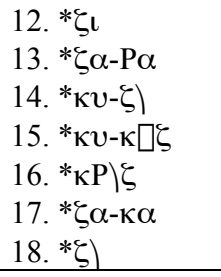 & $\begin{array}{l}\tau \iota \\
\mathrm{O}-\alpha \mathrm{P} \alpha \\
\kappa v \tau) \\
\kappa v \kappa \omega \square \varphi \\
\kappa \omega \alpha \varphi \\
\tau \alpha \kappa \alpha, \varphi \alpha \kappa \alpha \\
\tau)\end{array}$ & $\begin{array}{l}-\sigma \mathrm{l} \\
\sigma \alpha \alpha \mathrm{P} \alpha \\
\kappa v \sigma) \\
\kappa v \kappa о \varphi \\
\kappa \mathrm{HPo \varphi} \\
\sigma \alpha \alpha \kappa) \\
\sigma) \\
\end{array}$ & $\begin{array}{l}\text { 'osso' } \\
\text { 'asa, pluma' } \\
\text { ‘fogo' } \\
\text { 'macaco' } \\
\text { 'papagaio' } \\
\text { 'branco' } \\
\text { 'semente' }\end{array}$ \\
\hline$* x$ & $\begin{array}{l}\text { 19. } \quad{ }^{*} \chi \leftrightarrow P, \\
\chi \leftrightarrow \tau \\
\text { 20. }{ }^{*} \mu \mathrm{E} \chi \\
\text { 21. }{ }^{*} \kappa \alpha-\chi \omega \alpha \\
\text { 22. }{ }^{*} \chi \omega \alpha \\
\text { 23. }{ }^{*} \chi \alpha, \chi \alpha \mu\end{array}$ & $\begin{array}{l}\tau \Sigma \varepsilon \mathrm{P} \varepsilon \\
\mu \beta \mathrm{E} \tau \Sigma \mathrm{l}, \\
\mu \beta \mathrm{EP}, \\
\kappa \alpha \tau \omega \alpha \\
\tau \omega \alpha \\
\tau \alpha\end{array}$ & $\begin{array}{l}\sigma \varepsilon P \varepsilon \\
\mu E \tau- \\
\kappa \alpha \tau \omega \alpha \\
\tau \omega \alpha \\
\tau \alpha\end{array}$ & $\begin{array}{l}\text { 'queimar' } \\
\text { 'bom' } \\
\text { 'sal' } \\
\text { 'dente' } \\
\text { 'ficar.em.pé' }\end{array}$ \\
\hline$* \mu$ & 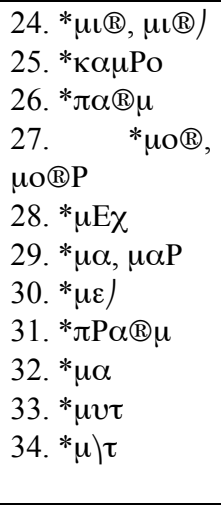 & 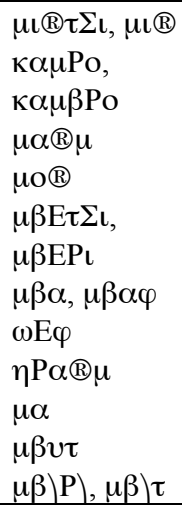 & $\begin{array}{l}\mu \iota \AA \\
\kappa H \alpha \alpha \mu \mathrm{Po} \\
\pi \alpha ® \mu \\
\mu \mathrm{\circledR} \\
\mu E \tau- \\
\mu \beta \alpha \\
\mu \varepsilon v \iota \\
\eta \mathrm{P} \alpha \AA \mu \\
\mu \beta \alpha, \mu \alpha \\
\mu v \tau v \\
\mu \mid \mathrm{Pl}\end{array}$ & $\begin{array}{l}\text { 'jacaré' } \\
\text { 'sangue' } \\
\text { 'pai' } \\
\text { ‘ir' } \\
\text { 'bom' } \\
\text { 'ouvir' } \\
\text { 'mel' } \\
\text { 'fome' } \\
\text { 'fígado' } \\
\text { 'pescoço' } \\
\text { 'sol' }\end{array}$ \\
\hline
\end{tabular}

Quado 2 - Dados histórico-comparativos

Fonte: Autoria própria. 
A partir dessa lista de cognatos foi possível levantar algumas hipóteses quanto às semelhanças e às diferenças do tapajúna em relação ao Proto-Jê e o que pode ter ocorrido nas formas mudadas.

O dado 1, por exemplo, mostra que o protofonema /*ª/, aparentemente, parece ser refletido como $/ \mathrm{h} /$ em tapajúna, havendo o surgimento de $/ \mathrm{w} /$ entre a consoante inicial e a vogal, ou seja, a consoante oclusiva bilabial /* $\mathrm{p} /$ pode ter passado a fricativa glotal e o que mantém o traço bilabial do protofonema é o aparente surgimento da aproximante bilabial /w/ (posteriormente discutiremos esse dado). Nos dados 4, 6 e 9, ocorre o mesmo processo, porém, em outros dados, quando o fonema seguinte à porto-forma não é vocálico, não há ocorrência da aproximante bilabial, o que nos leva a hipotetizar que o fonema / ${ }^{*} \mathrm{p} /$ é refletido como /h/, como se verifica nos dados 2 , 7 e 11 .

Há casos também em que /*p/ passa a /m/, isto é, sofre apenas nasalização, o que pode ser explicado, no caso do dado 3 ("pãm > mãm), pelo fato de a vogal nasal influenciar o fonema inicial. Tal explicação é plausível nesse dado, já no dado 4, em que a consoante seguinte é nasal, não ocorre esse fenômeno. Precisaríamos de mais dados para levantar a hipótese de que esse processo ocorre, quando a consoante seguinte à vogal é também nasal. Essa talvez essa seja uma das explicações possíveis para esse caso.

O dado 8 tem a mesma forma do dado 1 *pa (o que pode ser analisado como um caso de homonímia no Proto-Jê), cuja forma refletida no tapajúna é wa, diferentemente da forma do dado 1 cuja forma é hwa. O que há de diferente é a não presença do fonema /h/ no início da palavra, o contrário do que seria o esperado, pois, se olharmos para as outras ocorrências e para o próprio dado 1, veremos que a maioria das ocorrências mostra uma certa regularidade do surgimento de tal fonema como reflexo do protofonema /* p/. O mesmo fenômeno, ou seja, o apagamento ou o não surgimento da fricativa glotal ocorre no dado 10.

O que se observa nesses dados é apenas a manutenção do traço bilabial do protofonema inicial e, no caso do dado 8, o ambiente fonético-fonológico é o mesmo do dado 1, processo que abre caminho para muitas explicações, pois, se o ambiente fonético-fonológico é o mesmo, que fator (ou fatores) pode (ou podem) ter causado a diferença das formas sincrônicas do tapajúna? Talvez uma das hipóteses a ser levantada é o fato de, possivelmente, o fonema $/ \mathrm{h} /$ marcar um surgimento posterior e o que refletiria o protofonema seria a aproximante bilabial /w/. Essa seria uma das hipóteses a ser conferida, se a maioria dos dados não mostrasse o contrário, isto é, a maioria dos dados apresenta a ocorrência da fricativa glotal como reflexo do protofonema oclusivo bilabial surdo. Assim, podemos hipotetizar que, possivelmente, nesses dados em que a fricativa glotal não aparece trata-se de um estágio posterior de mudança. Isso quer dizer que é possível que a não ocorrência da fricativa glotal tenha ocorrido posteriormente, correspondendo a um 
último estágio de transformação pelo qual a língua passou. Verificamos, portanto, uma hipótese contrária à primeira levantada quanto a esse fenômeno.

No suyá, alguns dados são semelhantes aos do tapajúna no que se refere à ocorrência do protofonema /* $\mathrm{p} /$, tais como os dados 2, 4, 5, 6, 7, 9, 10 e 11. Vemos diferenças nos dados 1, 3 e 8 . O dado 1 não apresenta o fonema inicial /h/ que ocorre no tapajúna; os dados 3 e 8, por sua vez, mantêm a consoante oclusiva bilabial surda da protoforma.

Outro fonema que sofre alteração é o /z/ que, no tapajúna, sofre desvozeamento e muda o modo de articulação, passando a oclusiva alveolar surda /*t/, como nos dados 12 e 18 ( ${ }^{*} \mathrm{zi}>$ ti e ${ }^{*} \mathrm{zi}>$ tí, respectivamente), e mantendo o traço alveolar do protofonema.

Dos dados observados, o dado 13 apresenta a mudança de ${ }^{*} \mathrm{z} />\varnothing$, em que o protofonema inicial *z que, de acordo com Ribeiro (2004), corresponde à terceira pessoa, desaparece no tapajúna ( ${ }^{\star} z a-r a ~>\varnothing$-ara). Além desse fenômeno, em fronteira de palavra (considerando a porto-forma) $/{ }^{*} \mathrm{z} /$ muda para $/ \mathrm{j} /$, como podemos ver nos dados 15 e 16, em que a consoante fricativa alveolar sonora / ${ }^{*} \mathrm{z} /$ sofre palatalização e um certo afrouxamento, passando a aproximante palatal /j/.

No suyá, temos a ocorrência de /s/, no início de palavra ou de sílaba, como reflexo do protofonema / ${ }^{*} \mathrm{z} /$ nos dados $12,13,14,17$ e 18 já nos dados 15 e 16 , verificamos o mesmo fenômeno que ocorre no tapajúna, ou seja, a consoante fricativa alveolar sonora do Proto-Jê passa a aproximante palatal /j/.

Quanto ao protofonema /*c c/, podemos verificar em tapajúna que, nos dados 19 e 20, sofre alteração de oclusiva palatal surda no Proto-Jê para oclusiva alveolar africada surda /t $\mathrm{f} /$ e nos demais dados sofre alteração para oclusiva alveolar surda /t/, no tapajúna. No caso do dado 22, Ribeiro (2004) considera que o protofonema inicial corresponde ao morfema prefixo relacional, o qual, no tapajúna, parece já estar preso à raiz e não mais ser considerado prefixo relacional e sim um fonema integrante do radical. Considerando a porto-forma, em fronteira de palavra, como no dado $20,{ }^{*} \mathrm{c} /$ passa a /r/ antes da vogal /i/. No caso da variação, trata-se do sufixo $\left\{-\mathrm{t} \int \mathrm{i}\right\}$, que, entre outras coisas, pode indicar aumentativo, o que coincide em várias línguas Jê, como é o caso do Mebengôkre.

O suyá apresenta o dado 19 e o 20 com o fonema /s/ e o fonema /t/ refletindo o protofonema $/{ }^{*} \mathrm{c} /$, no início e em fronteira de palavra, respectivamente. Nos demais dados, verificamos semelhanças entre essa língua e o tapajúna.

Como podemos observar no quadro 2 , a consoante $/{ }^{*} \mathrm{~m} /$ sofre algumas alterações em alguns dados do tapajúna. Nos dados, 20, 29, 33 e 34, o protofonema $/{ }^{*} \mathrm{~m} /$ sofre pré-nasalização passando a /mb/. Na verdade, ocorre em tapajúna variação de faixa etária, porquanto os mais velhos ainda usam a consoante nasal 
e os mais jovens usam a pré-nasalizada. Contudo, tal fenômeno ainda está em estudo e ainda não se pode afirmar que a faixa etária seja o fator de influência para tal mudança ou se há outros fatores envolvidos nesse processo.

É possível também verificar a desnasalização e a mudança do modo de articulação no dado 30, em que /*m/ é refletido como aproximante bilabial /w/.

Nos dados 24, 25, 26, 27, 31 e 32, verificamos a não ocorrência de mudanças com relação ao protofonema, visto que a consoante $/{ }^{*} \mathrm{~m} /$ do Proto-Jê tem como reflexo o fonema $/ \mathrm{m} /$ nos diversos ambientes fonético-fonológicos, tais como: início de palavra, fronteira de sílaba e fronteira de palavra.

O suyá também apresenta as consoantes pré-nasalizadas como reflexo do protofonema $/{ }^{*} \mathrm{~m} /$, contudo, ocorrem em menor escala que no tapajúna. Os dados 29 e 32 do suyá confirmam tal afirmação. Nos demais dados, não há mudanças com relação à porto-forma.

A seguir, serão apresentadas as correspondências entre o tapajúna, o suyá e o proto-jê.

\section{Correspondências entre tapajúna, suyá e Proto-Jê}

As correspondências entre as consoantes do tapajúna e do suyá evidenciam o parentesco genético entre ambas as línguas e quão próximas estão na árvore do Tronco Macro-Jê, como é possível verificar nos dados já apresentados nos quadros 1 e 2. Sendo assim, no quadro 3 apresentamos as correspondências dos fonemas consonantais analisados e seus respectivos contextos fonético-fonológicos.

Nesse quadro é apresentada uma síntese do que foi observado no item anterior, demonstrando todos os ambientes fonético-fonológicos nos quais foram encontradas as consoantes analisadas. O que se observa é que, em relação aos dados do Proto-Jê, as duas línguas - tapajúna e suyá - mantêm quase todos os traços consonantais, somente em alguns contextos é que algumas consoantes sofrem alterações. Na maioria das vezes sofrem alterações somente em um traço como, por exemplo, uma consoante mudar apenas o traço de sonoridade ou apenas o ponto de articulação.

No caso das variações em mesmos contextos fonético-fonológicos, há a questão da variação de faixa etária, como a ocorrência das pré-nasalizadas na fala dos mais jovens do povo tapajúna, enquanto os mais velhos usam as nasais. Quanto às aspiradas ainda não se pode fazer nenhuma afirmação, haja vista ser necessário analisar um maior número de dados. 


\begin{tabular}{|c|c|c|}
\hline Protofonema & Tapajúna & Suyá \\
\hline$* \mu$ & 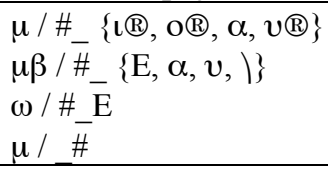 & $\begin{array}{l}\mu / \#_{-}\{\mathrm{l}, \mathrm{O} \circledast, \mathrm{E}, \varepsilon, \alpha, v, \\
1\} \\
\mu \beta / \mathrm{H}_{\alpha} \alpha \\
\mu /{ }^{\prime}\end{array}$ \\
\hline$* \pi$ & $\begin{array}{l}\eta / \#_{-}\{\omega, P\} \\
\mu / \#_{-} \leftrightarrow( \\
\omega / \#_{-}\{\alpha, 1\} \\
\pi / \# \\
\omega / E_{E}\end{array}$ & $\begin{array}{l}\omega / \#_{-}\{\alpha, 1\} \\
\pi / \#_{-}\{\alpha 囚, \alpha\} \\
\eta / \#_{-}\{\omega, P\} \\
\omega / E_{-} E\end{array}$ \\
\hline$* \xi$ & $\begin{array}{l}\tau / \#_{-}\{\alpha, \mathrm{l}, 1\} \\
\varphi / \#_{-} \alpha \\
\mathrm{O} / \#_{-} \alpha \\
\tau / V_{-} \mathrm{V} \\
\varphi /{ }_{-}\end{array}$ & $\begin{array}{l}\sigma / \#_{-}\{\alpha, \mathrm{l}, \mid\} \\
\sigma / V_{-} V \\
\varphi /{ }_{-}\end{array}$ \\
\hline$* \chi$ & $\begin{array}{l}\tau / \# \_\{\varepsilon, \omega, \alpha\} \\
\tau /)_{-} \iota \\
\tau / \alpha \_\omega \\
P / E_{-} \iota\end{array}$ & $\begin{array}{l}\sigma / \# \_\varepsilon \\
\tau / \#-\{\omega, \alpha\} \\
\tau / /)_{-} \iota \\
\tau / \alpha \_\omega \\
\tau /{ }_{-}\end{array}$ \\
\hline
\end{tabular}

Quadro 3 - Correspondências

Fonte: Autoria própria.

\section{Considerações finais}

Este trabalho apresenta uma análise histórico-comparativa referente aos segmentos consonantais, resultado de uma pesquisa que foi desenvolvida sobre a língua tapajúna na área de Linguística Histórica. Foram analisados cognatos entre o tapajúna e o suyá, nos quais foram verificadas semelhanças entre as duas línguas e o que mantiveram com relação ao Proto-Jê, enfocando ainda as mudanças ocorridas no decorrer dos tempos nas duas línguas.

As hipóteses aqui apresentadas não são definitivas. A análise de um maior número de dados poderá confirmá-las ou não. No entanto o objetivo de nosso trabalho é contribuir para o maior conhecimento de línguas Jê, com vistas à reconstrução do Tronco Macro-Jê, bem como para os estudos desenvolvidos na área de Linguística Histórica com dados de línguas indígenas brasileiras.

FERREIRA-SILVA, M. N.; RODRIGUES, C. K. C. Comparing Tapajúna and Suyá consonants. Alfa, Araraquara, v.55, n.2, p.601-611, 2011.

- ABSTRACT: This study investigates historical and comparative aspects of Tapajúna (Jê family) consonantal features, comparing cognates between Tapajúna and Suyá (also of the Jê family), in an effort to specify similarities and differences on the way to a reconstruction of the Macro-Jê stock. The comparative method was employed to detect correspondences 
between the languages. Data from Tapajúna and Suyá were compared to Proto-Jê data, as reconstructed by Davis (1966). There are few differences between Tapajúna and Suyá. Where there are variations in identical phonetico-phonologycal contexts, the question of speaker age arises, since, for example, pre-nasalized consonants occur in the speech of young people, whereas older Tapajúna speakers use only nasalized consonants.

- KEYWORDS: Consonant. Historical comparative method. Tapajúna. Suyá. Phonetic. Phonology. Cognates.

\section{REFERÊNCIAS}

CROWLEY, T. An introduction to historical linguistics. 3.ed. Oxford: Oxford University Press, 1997.

DAVIS, I. Comparative Jê phonology. Estudos Linguísticos: revista brasileira de linguística teórica e aplicada, São Paulo, v.1, n.2, p.10-24, 1966.

FARACO, C. A. Linguística histórica: uma introdução ao estudo da história das línguas. São Paulo: Ática, 1991.

GABA.S JUNIOR, N. Linguística histórica. In: MUSSALIM, F.; BENTES, A. C. (Org.). Introdução aos estudos linguísticos: domínios e fronteiras. 2.ed. São Paulo: Cortez, 2001. v.1. p.77-103.

RIBEIRO, E. R. Prefixos relacionais em Jê e Karajá: um estudo históricocomparativo. Liames, Campinas, n.4, p.91-101, 2004.

SANTOS, L. C. dos. Descrição dos aspectos morfossintáticos da língua Suyá (Kisêdjê) Família Jê. 1997. 178f. Tese (Doutorado em Linguística) - Instituto de Letras, Universidade Federal de Santa Catarina, Florianópolis. 1997.

SEKI, L. Evidências de relações genéticas na família Jê. SEMINÁRIO DO GEL, 36 ., 1989, Lorena. Anais... São Paulo: Grupo de Estudos Linguísticos, 1989. p.604-6011.

\section{BIBLIOGRAFIA CONSULTADA}

BENVENISTE, E. Problemas de linguística geral I. 4.ed. Campinas: Pontes, 1995.

CRYSTAL, D. Dicionário de linguística e fonética. Tradução e adaptação de Maria Carmelita Pádua Dias. Rio de Janeiro: Zahar, 2000.

FERREIRA, M. Aspectos fonético-fonológicos da língua tapajúna com vistas à elaboração de um sistema ortográfico: alguns problemas. Belém, 2006. Manuscrito.

. Descriptive studies of Tapajúna language. Belém, 2006. Manuscrito.

. Estudo morfossintático da língua parkatêjê. 2003. 276f. Tese (Doutorado em Linguística) -Instituto de Estudos da Linguagem, Universidade de Campinas, Campinas. 2003. 
HOCK, H. H. Principles of historical linguistics. Berlin: Mouton, 1986.

SEKI, L. O krenák (Botocudo/Borum) e línguas Jê. In: SANTOS, L., PONTES, I. (Org.). Línguas Jê, estudos vários. Londrina: Ed. da UEL, 2002. p.15-40.

Recebido em março de 2011.

Aprovado em maio de 2011. 
